\title{
Estandarización del Test de Dependencia al Celular para estudiantes universitarios de Arequipa
}

\author{
Katherine Gamero \\ Conni Flores \\ Universidad Nacional de San Agustín, Arequipa, Perú \\ Walter L. Arias \\ Karla D. Ceballos \\ Alessandra Román \\ Eduardo Marquina \\ Universidad Católica San Pablo, Arequipa, Perú
}

Recibido: 1 de diciembre del 2015/ Aprobado: 20 de abril del 2016

Se aportan evidencias de validez y confiabilidad del Test de Dependencia al Móvil, el cual consta de 22 items en una escala tipo Likert. El instrumento fue aplicado a dos muestras de estudiantes de una universidad pública $(N=1044)$ y otra privada $(N=356)$ de la ciudad de Arequipa. Para analizar su validez, se realizó un análisis factorial exploratorio. Se identificaron tres factores. El primer factor fue denominado abstinencia y tolerancia ( $\alpha=0,901)$; el segundo, abuso y dificultad para controlar el impulso $(\alpha=0,853)$, y el tercero, problemas ocasionados por el uso excesivo $(\alpha=0,762)$. Se crearon normas de administración y baremos en percentiles para estudiantes de la universidad pública y de la privada.

adicción al celular / dependencia / validez / confiabilidad

\section{A Standardized Mobile Phone Test on University Students in the City of Arequipa, Peru}

The objective of this study is to determine validity and reliability of the Mobile Phone Dependency Test (CPDT), in a 22 items Likert scale-based instrument. The Test was administered to two samples, a public university $(N=1044)$ and a private university $(N=356)$ in the Peruvian city of Arequipa. Validity was determined using an exploratory factor analysis. Three factors were identified: abstinence and tolerance $(\alpha=0,901)$, abuse and problems with impulse control $(\alpha=0,853)$, and problems with excessive mobile phone use $(\alpha=0,853)$. Additionally, percentile ranks were created for both public and private universities.

mobile phone addiction / psychometry / dependence / validity / reliability

Correo electrónico: warias@ucsp.edu.pe 


\section{INTRODUCCIÓN}

En los últimos años, tras la aparición de las nuevas tecnologías, se ha presentado un uso exagerado de las mismas que ha originado problemas de diversa índole en las áreas personal, laboral, familiar, académica y social de las personas. En este contexto, podemos señalar, al igual que Salas (2014), que ciertas adicciones se han vuelto cada vez más frecuentes en los últimos años. Entre estas, se puede mencionar la adicción a Internet, a las tecnologías, a los celulares, a las redes sociales, a los videojuegos, al trabajo, a los juegos de azar, al sexo, a las compras, etc. Como se ve, las adicciones tecnológicas, término acuñado por Griffiths (1995), están muy presentes a través de diversos medios y formas.

Sin embargo, como señalan Echeburúa y Corral (2010), la adicción a nuevas tecnologías viene señalada no tanto por la frecuencia de uso, sino por la relación de dependencia que crea, con pérdida de control del sujeto sobre su conducta y una importante interferencia en su vida cotidiana. En ese sentido, numerosos investigadores internacionales ya están dando la alerta de que muchas personas con celular están sufriendo problemas físicos y psicológicos como ansiedad, palpitaciones y sudoraciones, sobre todo cuando olvidan el celular en casa, cuando no cuentan con saldo, o se quedan sin cobertura de red o sin batería.

Así, el uso del celular de manera abusiva o dependiente es un problema actual y urgente porque cada día se incrementa, y tiene como agentes principales a los adolescentes, ya que es a partir de los 14 años que se ha reportado mayor susceptibilidad para volverse adicto al uso del celular (Chóliz y Villanueva, 2011). Los problemas de su uso excesivo o dependiente se derivan del hecho de que afecta, de manera incontrolada y silenciosa, todos los aspectos de la vida de los jóvenes, y se encubre bajo la apariencia de modas o necesidades de ocio (Lorente, 2002).

La adicción al celular es un trastorno conductual y/o psicológico que forma parte de los trastornos adictivos no convencionales y tecnológicos que, en la actualidad, están presentes en nuestro medio y en nuestra sociedad, y afectan con mayor intensidad a los jóvenes de ambos sexos (Bononato, 2005). La adicción al celular se adquiere como cualquier otro tipo de adicción, pues su uso se convierte en adicción cuando pasa a ser una conducta irreprimible, incontrolable, repetitiva, persistente y exagerada, que produce placer y genera una pérdida del control en el sujeto (Criado, 2005). Sin embargo, otros autores sugieren no usar la denominación de adicción en los casos de uso excesivo y dependiente de las tecnologías porque la variedad de usos de Internet y el celular no se circunscriben de manera exclusiva a la conducta adictiva, como sí ocurre con las sustancias psicoactivas (SPA), además de que su naturaleza es sincrónica y las tecnologías son más el medio que el fin, de manera que se puede considerar más adecuado decir que se es adicto al uso del chat, pero no al celular, o bien ser adicto a los videojuegos, pero no a la Internet (Carbonell, Fúster, Chamarro, y Osberst; y 2012; Carbonell, Chamarro, Griffiths, Oberst, Cladellas y Talarn, 2012). 
Aunque el tema es controversial, ya se ha empezado a hablar de que las nuevas tecnologías han pasado a formar parte de las denominadas adicciones psicológicas o adicciones sin drogas (Salas, 2014). En ese sentido, en un primer momento, el tema de la adicción a la Internet fue planteado por Goldberg en 1995 (Sandoz, 2004) y presentado por Young en 1996, lo que supuso trasladar los criterios diagnósticos de las adicciones a SPA al uso desmedido e incontrolable de la Internet (Sánchez-Carbonell, Beranuy, Castellana, Chamarro, y Oberst, 2008). Luego, se puso énfasis en la adicción al celular, ya que, dados los avances tecnológicos en la telefonía celular, esta subsume o abarca la adicción a Internet, porque los celulares, en la actualidad, cuentan con funciones multimedia, hipermedia e hipertexto que viabilizan el acceso instantáneo al mundo virtual con fines educativos, recreativos y de comunicación a través de sus múltiples aplicaciones.

Se debe aclarar, empero, que el uso del celular ha reportado tanto beneficios como efectos negativos en los jóvenes. En el primer caso, por ejemplo, se han implementado actividades de e-learning a través del celular (Pedrera y Revuelta, 2015), mientras que, en el segundo, son cada vez más constantes las formas de acoso virtual o cyberbullying a través del celular (Buelga, Cava, y Musitu, 2010). Como fuere, lo cierto es que el celular no es malo per se, sino que las personas hacemos un uso equivocado de este aparato. Lo mismo puede decirse de todas las otras formas de adicciones tecnológicas, como los videojuegos o la Internet (Labrador y Villadangos, 2010).
Ahora bien, diversos investigadores han comenzado a valorar y medir este fenómeno a través de escalas diseñadas especialmente para ello. Entre las más usadas en el habla anglosajona, podemos mencionar Mobile Phone Dependence Questionnaire, Scale of Problem Mobile Phone Use, Scale of Self-perception of Text-Message Dependence, Mobile Phone Problem Use Scale, Cell Phone Overuse Scale, Mobile Phone Addiction Scale, Mobile Phone Dependence Inventory, Excessive Cellular Phone Use Survey, Mobile Phone Addiction Index (Pedrero, Rodríguez, y Ruiz, 2012). En nuestro idioma, son los españoles quienes desde hace poco menos de veinte años vienen investigando el problema de la adicción al celular, y han creado diversos instrumentos de medición como la Escala de Medición del Uso Problemático del Móvil, Cuestionario de Experiencias relacionadas con el Móvil o la Prueba de Dependencia al Celular (Test of Mobile Phone Dependence), de Mariano Chóliz (2012).

Asimismo, mediante la aplicación de estos instrumentos, se ha podido determinar ciertos patrones de uso patológico o adictivo del celular, tales como su mayor prevalencia en grupos etarios a partir de los 15 años en adelante o en las mujeres (Chóliz, 2012; Chóliz y Villanueva, 2011; Chóliz, Villanueva, y Chóliz, 2009). Al respecto, se ha indicado que, mientras las mujeres usan el celular para fines sociales y de interacción con sus amigos y pares, los varones hacen uso del celular para jugar, buscar información o para probar aplicaciones tecnológicas (Carbonell, Chamarro, Griffiths, Oberst, Cladellas, y 
Talarn, 2012; Carbonell, Fúster, Chamarro, y Osberst, 2012). También se ha hecho mención al fenómeno fear of missing out o miedo a perderse algo, que se relaciona con la constante revisión del celular y envío de mensajes a través de la función WhatsApp (Barrio, 2014). Otros fenómenos relacionados con la adicción al celular son el cyberbullyng, el sexting, la nomofilia y la nomofobia (García y Fabila, 2014).

Como podemos darnos cuenta, son investigaciones internacionales las que nos proporcionan información acerca de este tema, que va tomando mucha importancia por los problemas que genera. Nuestro país, actualmente, se está viendo afectado por esta problemática, aunque no se han efectuado muchas investigaciones que aborden el tema por la carencia de instrumentos de exploración psicológica validados y normalizados para nuestro medio. Uno de los investigadores peruanos que aborda esta temática es Edwin Salas, quien viene investigando el uso adictivo de redes sociales en universitarios limeños (Escurra y Salas, 2014; Salas y Escurra, 2014), y otras adicciones comportamentales (Salas, 2014).

En Arequipa, el tema de la tecnología también está siendo investigado cada vez más con mayor interés, sobre todo en contextos educativos y laborales. Por ejemplo, Arias y Masías (2014) han reportado las diferencias acerca del ocio cibernético entre trabajadores de instituciones públicas y privadas; también se ha reportado el acceso a las Tecnologías de la Información (TIC) en colegios públicos y privados de Arequipa (Arias, 2015). Con respecto a las adicciones tecnológicas,
Quevedo y Ramírez (2005) realizaron el primer estudio sobre adicción a Internet de la región, mientras que la adicción al celular fue abordada por primera vez por Morales (2012) utilizando una muestra de 1253 estudiantes de una universidad privada de Arequipa, y encontró que el $8,1 \%$ de mujeres y el $3,4 \%$ de varones presentan síntomas de adicción al celular. Además, quienes tenían menor edad eran los que presentaban mayor cantidad de problemas relacionados con el uso del celular. La principal limitación de esta investigación es que se usó la Prueba de Adicción a Internet sin que esta hubiera sido estandarizada previamente.

Esto nos da cuenta de la importancia y la necesidad de contar con instrumentos validados para investigar los problemas derivados del uso de la tecnología en la región Arequipa. Por ello, mediante el presente artículo, se muestran los resultados del proceso de estandarización de la Prueba de Adicción al Celular de Mariano Chóliz (2012) en una muestra de estudiantes universitarios de la ciudad de Arequipa, considerando que los jóvenes son los más vulnerables a este tipo de dependencia, ya que se ven inmersos en un mundo tecnológicamente cambiante, donde el contar con un celular es necesario no solo como una herramienta de comunicación básica, sino que es indispensable y vital para llevar a cabo sus relaciones interpersonales y adquirir estatus entre sus pares (Forunati y Magnanelli, 2002; Muñoz-Rivas y Agustín, 2005). Sin embargo, se conoce también, por diferentes investigaciones, que el uso excesivo e inadecuado puede interferir en las áreas 
básicas de su desarrollo personal, así como centrar sus relaciones interpersonales en un aparato tecnológico y perder el contacto cara a cara con su entorno social (Becoña, 2001; Castellana, Sánchez-Carbonell, Graner, y Beranuy, 2007).

En términos psicométricos, dado que el presente estudio es de tipo instrumental, se entiende por estandarización el proceso mediante el cual se establecen procedimientos unívocos para la aplicación, calificación e interpretación de un test psicométrico (Aliaga, 2000). Para llevar a cabo este proceso, es necesario identificar las puntuaciones directas en la muestra, las cuales son convertidas en una calificación normativa que imparte información acerca de la ubicación de la puntuación de una prueba con respecto a otras puntuaciones de la misma prueba (Mora, 2013). La norma se constituye a partir de un grupo de personas, que representa a la población general a la cual se le aplicará la prueba, para derivar rangos de percentiles y calificaciones estándar.

Esto significa que una prueba dada podrá ser usada en una muestra amplia y representativa de la población previamente delimitada, cuyos resultados pueden considerarse válidos según los criterios de aplicación, calificación e interpretación. Por tanto, a partir de allí, una prueba estandarizada sirve para medir, de manera consistente en el tiempo, un fenómeno o variable para la cual fue construida, lo que supone obtener sus propiedades de validez y confiabilidad.

En ese sentido, la investigación científica hace uso de teorías, conceptos, metodologías, técnicas e instrumentos con el fin de aumentar el conocimiento existente (Bunge, 1989), pero ello implica utilizar instrumentos de medición válidos y confiables. Los instrumentos son, por tanto, aquellas herramientas que permiten obtener evidencia, y cuando esta se provee en términos de unidades de medida, entonces tal instrumento es catalogado como científico. Es por ello que estandarizar un instrumento ofrece criterios de calificación e interpretación de las puntuaciones bajo los mismos parámetros, por lo que se garantiza que los resultados son confiables. En ese sentido, la adicción al celular ha reportado valores tan disímiles, que van del 2,8\% hasta el $85 \%$ (Griffiths, 2013), que hace difícil comprender y caracterizar sus manifestaciones clínicas, por lo que es necesario contar con instrumentos validados para muestras representativas en cada región.

\section{Método}

\section{Participantes}

Se trabajó con una muestra de 1044 estudiantes de la Universidad Nacional San Agustín y 356 estudiantes de la Universidad Católica San Pablo; se utilizaron como criterios de evaluación el que sean estudiantes universitarios matriculados de primero a décimo segundo ciclo de estudios (según la carrera profesional) de las diferentes carreras profesionales de cada universidad y que cuenten con celular. Se realizó un muestreo probabilístico estratificado según la población estudiantil en cada carrera de las dos universidades (Hernández, Fernández 
y Baptista, 2003). Las edades de los estudiantes fluctúan entre los 16 y los 33 años, con una media de 20,7 años y una desviación estándar de \pm 2.65 . El $50,14 \%$ de la muestra está conformado por varones, y el $49,86 \%$ por mujeres.

\section{Instrumento}

Se utilizó como instrumento de investigación el Test de Dependencia al Móvil (TDM), cuyo autor es Mariano Chóliz Montañés (Chóliz, 2012; Chóliz y Villanueva, 2010; Chóliz, Villanueva, y Chóliz, 2009). Esta prueba cuenta con cuatro factores planteados por el autor y obtenidos estadísticamente mediante análisis factorial. Los factores son abstinencia (ítems 13, 15, 20, 21 y 22), abuso y dificultad en controlar el impulso (items $1,4,5,6,8,9,11,16$ y 18), problemas ocasionados por el uso excesivo (ítems 2, 3,7 y 10) y tolerancia (ítems 12, 14, 17 y 19). Es importante mencionar que el autor viene trabajando con este mismo instrumento en la Universidad de San Marín de Porres, donde se realizó la adaptación lingüística correspondiente (ver Anexo 1).

\section{Procedimiento}

Para realizar la presente investigación psicométrica, se gestionaron los permisos correspondientes con las autoridades competentes en cuatro universidades de la ciudad de Arequipa, pero la respuesta fue positiva solo en dos universidades. Como siguiente paso, se coordinó con los profesores de curso de cada una de las áreas académicas con la finalidad de aplicar el Test de Dependencia al Móvil en una sola sesión; según la disponibilidad de tiempo y colaboración del docente, se procedió a la aplicación. Una vez en el aula, se les dio instrucciones generales a los alumnos participantes y se les motivó para que colaborasen con el llenado del cuestionario de manera veraz. El tiempo promedio de resolución fue de 10 minutos, lapso durante el cual se permaneció en el aula con la finalidad de absolver algunas dudas que tuviesen los alumnos. Después de culminar esta fase, se prosiguió con el procesamiento estadístico de los datos con el programa SPSS.

\section{Resultados}

Para medir la validez, se realizó un análisis descriptivo y correlacional con el fin de valorar la homogeneidad de los reactivos, lo cual implicó realizar correlaciones ítem-test mediante el coeficiente de correlación de Pearson.

Como puede observarse en la Tabla 1 , los promedios de los diferentes ítems son similares entre sí, salvo el promedio del ítem $8(M=2,35)$, que es un tanto mayor que el resto de ítems. Los estadísticos de asimetría y curtosis son, en general, adecuados, lo que demuestra que los puntajes de los diversos ítems no están sesgados y nos permitirán análisis confiables.

Por otro lado, en la Tabla 2, se aprecia una matriz donde las correlaciones ítemtest son todas positivas, con valores que oscilan entre 0.499 y 0.722 , lo cual implica que todos los ítems miden el mismo constructo. Asimismo, con la finalidad de valorar la estructura de la prueba y determinar su validez de constructo, se 
Tabla 1

Estadísticos descriptivos

\begin{tabular}{lllllll}
\hline & $M$ & $D E$ & Asimetría & $A S(D t)$ & Curtosis & $C U(D t)$ \\
\hline Ítem 1 & 1.26 & 1.157 & .667 & .065 & -.316 & .131 \\
Ítem 2 & 1.01 & 1.156 & .917 & .065 & -.122 & .131 \\
Ítem 3 & 0.69 & 0.986 & 1.431 & .065 & 1.382 & .131 \\
Ítem 4 & 1.32 & 1.103 & .573 & .065 & -.378 & .131 \\
Ítem 5 & 1.43 & 1.182 & .531 & .065 & -.536 & .131 \\
Ítem 6 & 1.72 & 1.275 & .303 & .065 & -.907 & .131 \\
Ítem 7 & 1.15 & 1.122 & .812 & .065 & -.091 & .131 \\
Ítem 8 & 2.35 & 1.222 & -.237 & .065 & -.936 & .131 \\
Ítem 9 & 1.45 & 1.108 & .564 & .065 & -.328 & .131 \\
Ítem 10 & 0.73 & 1.029 & 1.443 & .065 & 1.448 & .131 \\
Ítem 11 & 1.60 & 1.252 & .252 & .065 & -1.001 & .131 \\
Ítem 12 & 1.95 & 1.234 & -.021 & .065 & -.981 & .131 \\
Ítem 13 & 1.43 & 1.247 & .446 & .065 & -.861 & .131 \\
Ítem 14 & 1.57 & 1.182 & .264 & .065 & -.894 & .131 \\
Ítem 15 & 1.19 & 1.166 & .675 & .065 & -.506 & .131 \\
Ítem 16 & 1.32 & 1.203 & .590 & .065 & -.634 & .131 \\
Ítem 17 & 1.25 & 1.157 & .588 & .065 & -.555 & .131 \\
Ítem 18 & 1.78 & 1.364 & .168 & .065 & -1.219 & .131 \\
Ítem 19 & 1.36 & 1.264 & .486 & .065 & -.898 & .131 \\
Ítem 20 & 1.45 & 1.321 & .486 & .065 & -.926 & .131 \\
Ítem 21 & 1.69 & 1.310 & .167 & .065 & -1.143 & .131 \\
Ítem 22 & 1.17 & 1.218 & .730 & .065 & -.515 & .131 \\
\hline
\end{tabular}

realizó un análisis factorial, para lo cual se obtuvo el valor KMO y la prueba de esfericidad de Bartlett.

El contraste de esfericidad de Bartlett $\left(\mathrm{X}_{(231)}^{2}=14261,801 ; p<0,001\right)$ reveló que podemos rechazar la hipótesis nula de que las variables utilizadas en el análisis no se correlacionan en la población de la que hemos extraído la muestra, lo cual nos permite considerar la matriz de correlaciones adecuada para la factorización. Asimismo, la medida de adecuación muestral de Kaiser-Meyer-Olkin $(\mathrm{KMO}=0,949)$ también nos indica que la matriz de correlaciones es adecuada para el análisis.

Para análizar de la estructura del cuestionario, se empleó la técnica del análisis factorial exploratorio. El método de extracción de los factores ha sido el de Factorización por ejes principales, y para 


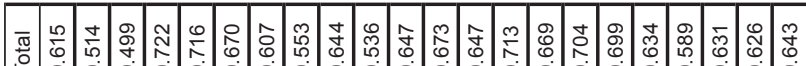

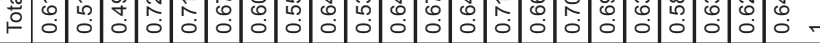

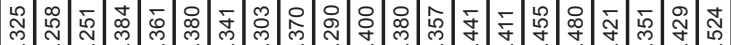

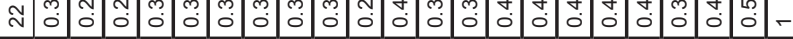

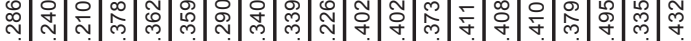

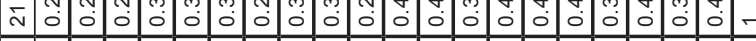

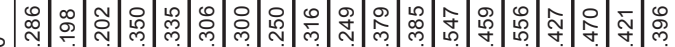

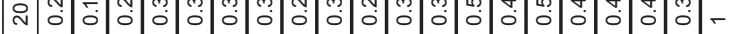

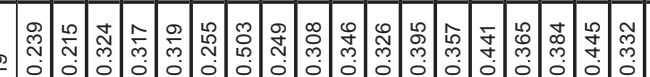

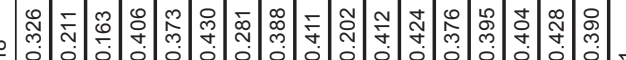

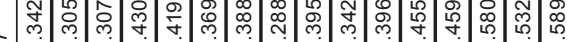

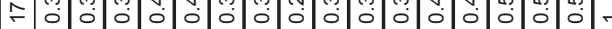

戹岱 F

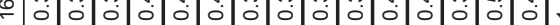

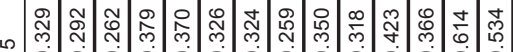

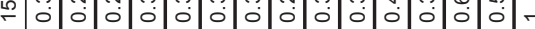

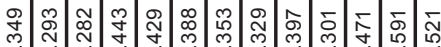

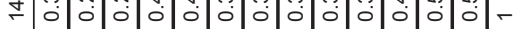

స్లి

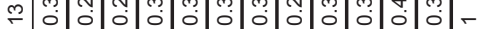

N

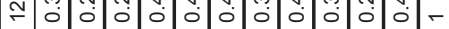

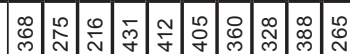

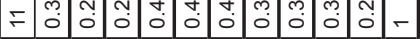

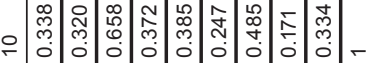

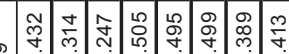

$\begin{array}{llllllllll}8 & 0 & 0 & 0 & 0 & 0 & 0 & 0 & \vdots & 0\end{array}$

更莳

$\begin{array}{llllllllllll}0 & 0 & 0 & 0 & 0 & 0 & 0 & 0 & 0 & 0\end{array}$

ల్లి స్లి

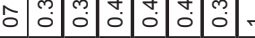

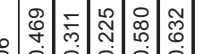

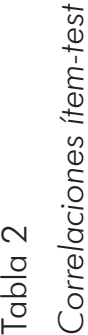

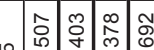

ᄂ

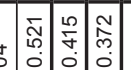

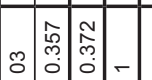

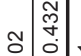

ธ - 
Tabla 3

KMO y Prueba de Bartlett

\begin{tabular}{|c|c|c|}
\hline $\begin{array}{l}\text { Medida de adecuación muestral de } \\
\text { Kaiser-Meyer-Olkin. }\end{array}$ & & .949 \\
\hline \multirow[t]{3}{*}{ Prueba de esfericidad de Bartlett } & $\begin{array}{l}\text { Chi- } \\
\text { cuadrado }\end{array}$ & 14261.801 \\
\hline & $\mathrm{Gl}$ & 231 \\
\hline & Sig. & .000 \\
\hline
\end{tabular}

realizar la rotación se ha empleado el método de Normalización de Promax, con un valor de Kappa $=4$. Se ha empleado esta técnica de rotación porque se asumió que existe una correlación entre los factores, ya que las dimensiones de las que consta el constructo (adicción al teléfono móvil) no son independientes entre sí.

Del análisis factorial, se extrajeron tres factores que explican el 54,74\% de la varianza. El primer factor explica el 40,7 $\%$ de la varianza, su autovalor es 8,95. Está compuesto por los ítems 11, 12, 13, $14,15,16,17,18,19,20,21$ y 22 . Se le ha denominado tolerancia y abstinencia, ya que hace referencia a los dos criterios más relevantes de la dependencia. Tolerancia se manifiesta por el hecho de que cada vez se utiliza el celular durante más tiempo para conseguir los mismos objetivos que al principio. Por otro lado, abstinencia se caracteriza por el malestar cuando no es posible tener el celular o si este no funciona adecuadamente. Dicho malestar puede manifestarse mediante intranquilidad, aburrimiento excesivo, dificultad de concentración en otras actividades, o incluso irritabilidad.
El segundo factor explica el 7,39\% de la varianza, su autovalor es 1,62 y está compuesto por los ítems 1, 2, 4, 5, 6, 8 y 9 del TDM, atendiendo al contenido de los ítems; este segundo factor se ha denominado abuso y dificultad para controlar el impulso, ya que se refiere a una utilización excesiva del móvil que se lleva a cabo en cualquier momento y situación, y a una gran dificultad para dejar de utilizarlo. El tercer factor explica el 6,64\% de la varianza, su autovalor es 1,46 y está conformado por los ítems 3, 7 y 10. Se le ha denominado problemas ocasionados por el uso excesivo, ya que estos ítems describen las consecuencias negativas de la utilización excesiva o inapropiada del móvil (ver Tabla 4 y 5).

Se ha considerado, para la exclusión de ítems, una saturación en el factor inferior a 0,30 , pero no hubo la necesidad de eliminar algún ítem, pues todos tuvieron una buena saturación. En la Tabla 6, podemos observar que las correlaciones entre los tres factores son directas, lo cual coincide con lo propuesto sobre el constructo adicción al celular, ya que las diferentes dimensiones de este deben 
Tabla 4

Varianza total explicada

\begin{tabular}{ccccc}
\hline & \multicolumn{2}{c}{ Autovalores iniciales } & \multicolumn{2}{c}{$\begin{array}{c}\text { Sumas de las saturaciones al } \\
\text { cuadrado de la extracción }\end{array}$} \\
\hline Total & $\begin{array}{c}\text { \% de la } \\
\text { varianza }\end{array}$ & $\%$ acumulado & Total & $\begin{array}{c}\text { \% de la } \\
\text { varianza }\end{array}$ \\
\hline 8.956 & 40.708 & 40.708 & 8.446 & 38.392 \\
1.627 & 7.395 & 48.103 & 1.163 & 5.286 \\
1.462 & 6.644 & 54.747 & .991 & 4.502 \\
\hline
\end{tabular}

Método de extracción: Factorización de ejes principales

estar relacionadas entre sí de forma tal que, al incrementarse alguna de estas, las otras dimensiones también deben aumentar su valor.

Luego se procedió a realizar un análisis factorial confirmatorio (AFC) de la escala, la cual arroja índices de bondad de ajuste que nos indican que el modelo es aceptable, ya que los índices GFI y CFI son cercanos a 0,90 , mientras que el índice RMSEA es un poco mayor de 0,05 ; pero los índices RMR y SRMR son los esperados, ya que son menores de 0,05 (Pérez-Gil, Chacón y Moreno, 2000). Por ello, podemos concluir que el Test de Dependencia al Celular y sus tres subescalas conforman un instrumento válido para medir este constructo en nuestro medio.

Respecto a la fiabilidad de la prueba, la evaluamos por el método de consistencia interna mediante el coeficiente alpha de Cronbach. Encontramos que, en general, el Test de Dependencia al
Celular tiene altos niveles de confiabilidad: $\alpha=0,929$; respecto a sus factores, estos obtienen muy buenos niveles de confiabilidad: primer factor, $\alpha=0,901$; segundo factor, $\alpha=0,853$, y el tercer factor, $\alpha=0,762$, lo que nos indica que la prueba es confiable en nuestro medio y arrojará resultados fiables.

Finalmente, se establecieron los baremos con los respectivos percentiles para los valores obtenidos. Estos baremos generaron tres rangos de respuestas para la Dependencia al celular. Un nivel bajo del Test de Dependencia al Móvil o Celular se ubica entre los percentiles 0 y 25 ; un nivel moderado, entre los percentiles 30 y 70 ; un nivel alto, entre los percentiles 75 y 95 . Se han obtenido, además, estos valores según se trate de una universidad pública (Universidad Nacional de San Agustín) o privada (Universidad Católica San Pablo) (ver Anexo 2 y 3). 
Tabla 5

Matriz de configuración

\begin{tabular}{|c|c|c|c|}
\hline & \multicolumn{3}{|c|}{ Factor } \\
\hline & 1 & 2 & 3 \\
\hline Ítem 1 & & .560 & \\
\hline Ítem 2 & & .338 & \\
\hline Ítem 3 & & & .833 \\
\hline Ítem 4 & & .725 & \\
\hline Ítem 5 & & .759 & \\
\hline Ítem 6 & & .852 & \\
\hline Ítem 7 & & & .421 \\
\hline Ítem 8 & & .643 & \\
\hline Ítem 9 & & .552 & \\
\hline Ítem 10 & & & .792 \\
\hline Ítem 11 & .420 & & \\
\hline Ítem 12 & .414 & & \\
\hline Ítem 13 & .701 & & \\
\hline Ítem 14 & .680 & & \\
\hline Ítem 15 & .792 & & \\
\hline Ítem 16 & .473 & & \\
\hline Ítem 17 & .655 & & \\
\hline Ítem 18 & .467 & & \\
\hline Ítem 19 & .502 & & \\
\hline Ítem 20 & .802 & & \\
\hline Ítem 21 & .534 & & \\
\hline Ítem 22 & .526 & & \\
\hline
\end{tabular}

Nota: ${ }^{* *} \mathrm{p}<.001$

Tabla 6

Correlaciones entre factores

\begin{tabular}{llll}
\hline Factor & 1 & 2 & 3 \\
\hline 1 & - & & \\
2 & $.696^{* * *}$ & - & - \\
3 & $.501^{* * *}$ & $.519^{* * *}$ & - \\
\hline
\end{tabular}




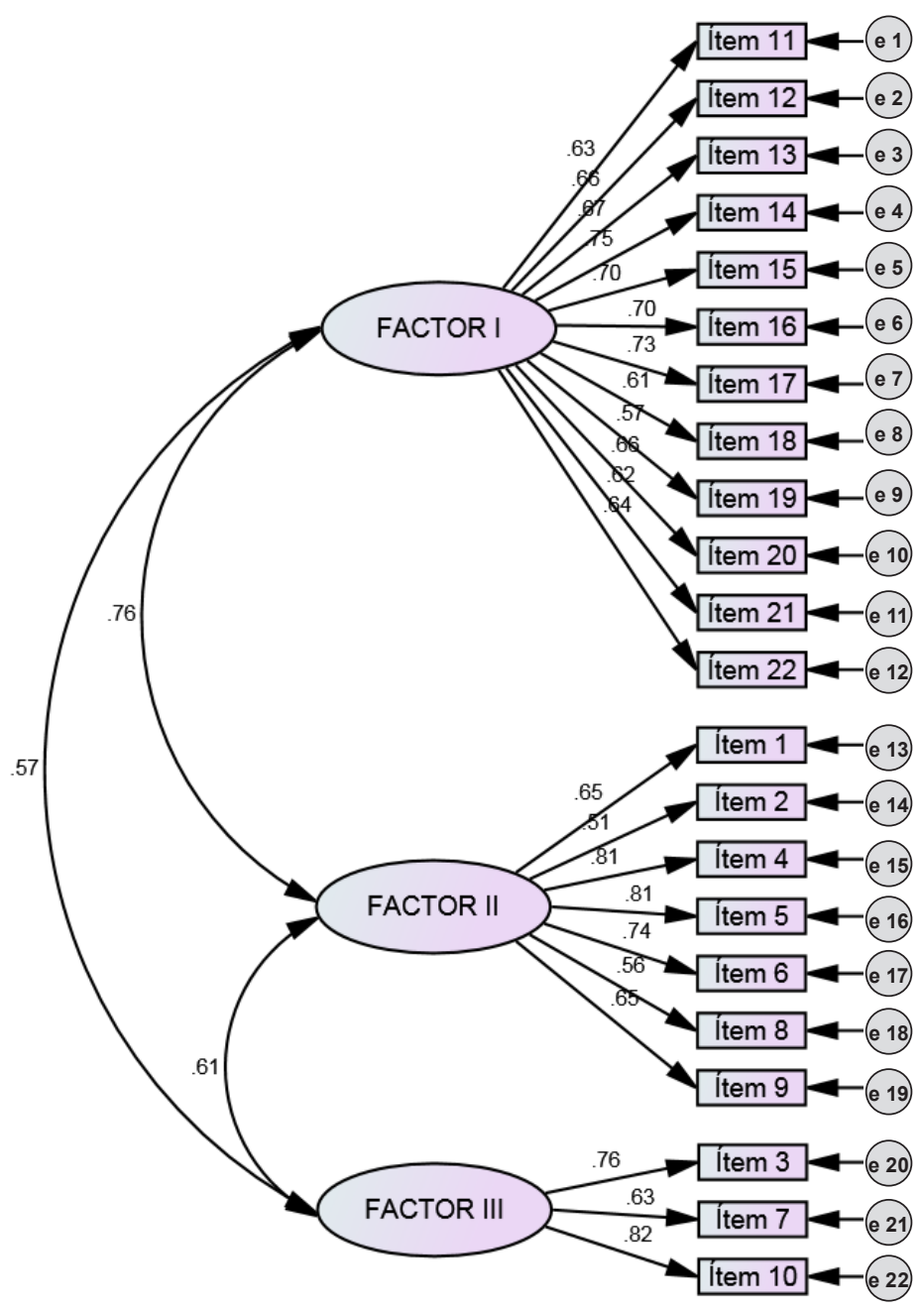

Figura 1. Análisis factorial confirmatorio 
Tabla 7

Índices de ajuste de bondad de la escala

\begin{tabular}{lc}
\hline \hline \multicolumn{1}{c}{ Estadísticos } & Adicción al celular \\
\hline X2 de bondad de ajuste & $186.60^{* *}$ \\
GI & 231 \\
GFI & 0.873 \\
CFI & 0.896 \\
RMR & 0.033 \\
RMSEA & 0.071 \\
SRMR & 0.021 \\
\hline
\end{tabular}

Nota: ${ }^{* *} p<.01$

\section{DISCUSIÓN}

En el presente estudio, se exponen los criterios para la estandarización del Test de Dependencia al Móvil de Mariano Chóliz (2012) en una muestra de 1400 estudiantes universitarios de la ciudad de Arequipa, provenientes de la Universidad Nacional de San Agustín (de gestión pública) y de la Universidad Católica San Pablo (de gestión privada). Los análisis de datos, efectuados inicialmente bajo el contexto de la teoría clásica de los tests (Abad, 2006), se realizaron con el nivel de análisis de ítems, y la confiabilidad por consistencia interna de cada una de las dimensiones ha permitido observar que las correlaciones ítem-test superaron el criterio propuesto por Kline (1998); respecto a la confiabilidad de la prueba, utilizamos el método de consistencia interna mediante el coeficiente alpha de Cronbach. Encontramos que, en general, el instrumento tiene altos niveles de confiabilidad: $\alpha=0,929$. Con respecto a sus factores, estos obtienen adecuados niveles de confiabilidad (primer factor, $\alpha=0,901$; segundo factor, $\alpha=0,853$, y tercer factor, $\alpha=0,762)$; la prueba es válida y confiable para la población universitaria de nuestro medio.

También se analizó la validez de constructo, para lo cual se obtuvo el valor KMO de 0.949, y la Prueba de Esfericidad de Bartlett arrojó un valor chi cuadrado de 14261.801, con 231 grados de libertad $\mathrm{y}$ un nivel de significancia de $\mathrm{p}<0.001$, por lo que, al realizar el análisis factorial, se obtuvieron tres factores. El primer factor explica el 40,7 \% de la varianza y está compuesto por 12 ítems $(11,12,13,14,15$, $16,17,18,19,20,21$ y 22). Se le ha denominado tolerancia y abstinencia, ya que agrupa los ítems de los factores tolerancia y abstinencia de la prueba de Chóliz. El segundo factor explica el 7,39 \% de la varianza y está compuesto por 7 ítems (1, $2,4,5,6,8$ y 9). Atendiendo al contenido de los ítems, este segundo factor se ha denominado abuso y dificultad para controlar el impulso, mientras que el tercer factor explica el 6,64 \% de la varianza y está conformado por 3 ítems (3, 7 y 10). 
Se le ha denominado problemas ocasionados por el uso excesivo. Es importante mencionar que se ha considerado, para la exclusión de ítems, una saturación en el factor inferior a 0,30 ; pero no hubo la necesidad de eliminar algún ítem, pues todos tuvieron una buena saturación.

Como se evidencia después del análisis factorial, se han obtenido tres factores, hecho que marca diferencias con la propuesta original del autor, que da cuenta de cuatro. Es importante destacar que los primeros estudios de la adicción al móvil llevados a cabo por Chóliz (2010) se realizaron cuando no era muy frecuente, como ahora, la conexión a Internet a través del móvil, y todavía no se había generalizado el uso de los smartphones. El móvil tenía numerosas funciones tecnológicas (grabación y reproducción de audio y video, fotografía, videojuegos, etc.), pero las principales herramientas de comunicación eran las llamadas y los mensajes de texto, o SMS. Esto explica que la versión inicial del Test de Dependencia al Móvil con 22 ítems se base, principalmente, en evaluar el uso de llamadas y mensajes de texto, y quede conformada por tres factores. Uno de los trabajos de investigación en el que fue utilizado este instrumento corresponde al estudio de Chóliz y Villanueva (2011), en el que participaron 1944 estudiantes de enseñanza secundaria obligatoria (ESO) y bachillerato, con edades comprendidas entre los 12 y 18 años en la provincia de Valencia (España).

En la actualidad, se utiliza principalmente Internet en el celular para los formatos de comunicación vía WhatsApp, Line, etc.; se utilizan mucho menos mensajes de texto o llamadas de voz. Esto obligó al autor a realizar pequeñas modificaciones en el test para adaptarlo a la nueva realidad, motivo por el cual en los ítems en los que aparece la palabra $S M S$, por ejemplo, se añadiera también la palabra WhatsApp. En realidad, el contenido del ítem no se modifica, en el sentido de que se refiere al mismo proceso psicológico (tolerancia, abstinencia, etc.), con independencia de la aplicación tecnológica que se esté usando.

Se utilizó, por lo tanto, la misma prueba de 22 ítems inicialmente diseñada, pero se evaluó solamente a personas que utilizaban mensajería de Internet mediante el móvil en una muestra de tipificación que estuvo constituida por 619 personas (235 varones y 384 mujeres) de edades comprendidas entre los 14 y 30 años, que fueron seleccionadas de forma aleatoria de entre aquellas personas que utilizan habitualmente las herramientas de comunicación del móvil, principalmente WhatsApp. Dentro de los resultados encontrados, y por medio del análisis factorial, se extrajeron cuatro factores denominados abstinencia, abuso y dificultad para controlar el impulso, problemas ocasionados por el uso excesivo y tolerancia, los cuales explican el $59,88 \%$ de la varianza.

Según las consideraciones del autor de la prueba, el TDM evalúa las principales dimensiones de la adicción y no tanto las particularidades de uso; el cuestionario es el mismo que el TDM inicial, pero la agrupación de los elementos es diferente, $\mathrm{y}$ conforman cuatro dimensiones en lugar de las tres de la versión anterior (Chóliz, 2012; 
Chóliz y Villanueva, 2010; Chóliz, Villanueva y Chóliz, 2009), que se justifican debido a las diferencias comportamentales entre el uso del móvil como teléfono y para enviar SMS, del uso del móvil como teléfono inteligente (Smartphone). Es por ello que los problemas de adicción también se manifiestan de forma distinta, principalmente a causa de la utilización de las herramientas de Internet.

Con respecto al análisis factorial realizado en nuestra investigación, podemos mencionar la diferencia en cuanto a la cantidad de factores hallados; se nota que, con una muestra española, los ítems se agrupan en cuatro factores, mientras que, en la muestra de Arequipa, estos mismos ítems se agrupan en tres. Nosotros presumimos que estas diferencias se explican debido a las características culturales, los avances tecnológicos y diferencias comportamentales en el uso del celular. Este se ha incrementado en todo el mundo debido a sus beneficios para la comunicación, y llega a introducirse incluso en los sectores económicamente menos favorecidos (Vargas-Hernández, 2014); sin embargo, es posible diferenciar tasas de adicción al celular según el país o de un estudio a otro. Griffiths (2013) comenta, por ejemplo, que las cifras son tan disímiles que van de 2,5 hasta el $85 \%$ de tasas de adicción al celular. En ese sentido, estas brechas se relacionan con el uso de diferentes instrumentos, o de dudosa procedencia, al no contar con criterios de validez y confiabilidad.

En Arequipa, por ejemplo, Morales (2012) abordó el tema por primera vez, pero utilizando la prueba de Young, que valora la adicción a Internet. El autor tuvo que adecuar el instrumento de Young para evaluar la adicción al celular en 1253 estudiantes de primer año de la Universidad Alas Peruanas. Es por ello que el presente estudio hace un aporte psicométrico relevante al presentar la versión estandarizada para estudiantes universitarios de Arequipa, con lo que nuevas investigaciones podrán llevarse a cabo bajo criterios de validez y confiabilidad adecuados para nuestra ciudad.

En ese sentido, el uso de las tecnologías ha abierto un nuevo campo de estudio para la psicología a través del uso adictivo o patológico de los diversos medios de comunicación como Internet o el teléfono celular (Saldaña, 2001). Si bien es cierto España es el país donde se ha desarrollado desde hace casi veinte años una gran cantidad de literatura sobre el tema (Adès y Lejoyeux, 2003; Alonso-Fernández, 2003; Oliva, Hidalgo, Moreno, Jiménez, Jiménez, Antolín, y Ramos, 2012), y se han construido varios instrumentos psicométricos para evaluar la adicción al celular (Beranuy, Chamarro, Graner, y Carbonell, 2009; Chóliz, 2012), en Arequipa, el uso de las tecnologías cobra cada vez mayor relevancia para los psicólogos, por cuanto se están realizando nuevos estudios sobre adicción a Internet, el cibersexo, el ocio cibernético, el burnout en teletrabajadores, así como estudios que buscan relacionar el uso de la computadora con la personalidad o con la salud ocupacional. En un estudio reciente que valora lo que piensan los estudiantes de últimos años de psicología de cuatro universidades de Arequipa sobre 
cuáles son los temas de interés en el futuro, las nuevas tecnologías son ubicadas en primer lugar por los estudiantes de la Universidad Nacional de San Agustín (Arias, Arista, Choque, Angles, Chávez, y Herrera, 2015).

Todo ello da cuenta de la relevancia que tiene el tema para los psicólogos de la región sur del país. Se espera que, con los baremos ofrecidos, fruto de la validación y estandarización del Test de Dependencia al Celular, se pueda profundizar en el conocimiento del tema con futuras investigaciones. De este modo, esperamos que este instrumento contribuya a un mayor conocimiento de esta problemática.

\section{RefERENCIAS}

Abad, F. (2006). Introducción a la psicometría: teoría clásica de los tests y teoría de la respuesta al ítem. España: S/E.

Adès, J., y Lejoyeux, M. (2003). Las nuevas adicciones: internet, sexo, deporte, compras, trabajo, dinero. Barcelona: Kairós.

Aliaga, T. (2000) Psicometría: tests psicométricos, confiabilidad y validez. Perú: S/E.

Alonso-Fernández, F. (2003). Las nuevas adicciones. Madrid: TEA.

Arias, W. L. (2014). Tecnologías de la información y la comunicación en colegios públicos y privados de Arequipa. Interacciones, 1(1), 11-28.

Arias, W. L., y Masías, M. A. (2015). Ocio cibernético en trabajadores de instituciones públicas y privadas de Arequipa. Ciencia y Trabajo, 16(50), 88-92.
Arias, W. L., Arista, M., Choque, M., Angles, U., Chávez, P., y Herrera, B. (2015). Una encuesta acerca de los psicólogos más representativos y el futuro de la psicología en Arequipa. Liberabit, 21(1), 123-139.

Barrio, A. (2014). Los adolescentes y el uso de los teléfonos móviles y de video juegos. International Journal of Developmental and Educational Psychology, 3(1), 563-570.

Becoña, E. (2001). Tratamiento psicológico de las conductas adictivas. Vigo: Nova Galicia Edicións.

Beranuy, M., Chamarro, A., Graner, C., y Carbonell, X. (2009). Validación de dos escalas breves para evaluar la adicción a Internet y el abuso del móvil. Psicothema, 21(3), 480-485.

Bononato, L. B. (2005). Adicciones y nuevas tecnologías. Proyecto Hombre, 55, 17-21.

Buelga, S., Cava, M. J., y Musitu, G. (2010). Cyberbullying: victimización entre adolescentes a través del teléfono móvil y de Internet. Psicothema, 22(4), 784-789.

Bunge, M. (1989). Ciencia y desarrollo. Buenos Aires: Siglo XXI.

Carbonell, X., Fúster, H., Chamarro, A., y Oberst, U. (2012). Adicción a Internet y móvil: una revisión de estudios empíricos españoles. Papeles del Psicólogo, 33(2), 82-89.

Carbonell, X., Chamarro, A., Griffiths, M. D., Oberst, U., Cladellas, R., y Talarn, A. (2012). Problematic Internet and Cellphone Use in Spanish 
Teenagers and Young Students. Anales de Psicología, 28(3), 789-796.

Castellana, M., Sánchez-Carbonell, X., Graner, C., y Beranuy, M. (2007). El adolescente ante las tecnologías de la información y la comunicación: internet, móvil y videojuegos. Papeles del Psicólogo, 28(3), 196-204.

Chóliz, M. (2012). Mobile-phone Addiction in Adolescence: The Test of Mobile Phone Dependence (TMD). Prog Health Sci, 2(1), 33-43.

Chóliz, M., y Villanueva, V. (2011). Evaluación de la adicción al móvil en la adolescencia. Revista Española de Drogodependencias, 36(2), 165-184.

Chóliz, M., Villanueva, V., y Chóliz, M. C. (2009). Ellas, ellos y su móvil: uso, abuso (¿y dependencia?) del teléfono móvil en la adolescencia. Revista Española de Drogodependencias, 34(1), 74-88.

Criado, M. A. (2005). Enfermos del móvil. Recuperado de http://www.el-mundo. es/ ariadna/2005/218/1106327395.html

Echeburúa, E., y Corral, P. (2010). Adicción a las nuevas tecnologías y a las redes sociales en jóvenes: un nuevo reto. Adicciones, 22(2), 91-96.

Escurra, L. M., y Salas, E. (2014). Construcción y validación del Cuestionario de Adicción a Redes Sociales (ARS). Liberabit, 20(1), 73-91.

García, V., y Fabila, A. M. (2014). Nomofilia vs. nomofobia. Irrupción del teléfono móvil en las dimensiones de vida de los jóvenes. Un tema pendiente para los estudios en comunicación.
Razón y Palabra, 86(2). Recuperado de www.razonypalabra.org.mx

Griffiths, M. D. (1995). Technological addictions. Clinical Psychology Forum, 76, 14-19.

Griffiths, M. D. (2013). Adolescent Mobile Phone Addiction: A Cause for Concern? Education and Health, 31(3), 76-78.

Forunati, L., y Magnanelli, A. M. (2002). El teléfono móvil de los jóvenes. Estudios de Juventud, 57(2), 59-78.

Hernández, R., Fernández, C., y Baptista, P. (2003). Metodología de la investigación (3. ${ }^{\mathrm{a}}$ ed.). México D.F.: McGraw-Hill.

Kline, P. (1998). The New Psychometrics: Science, Psychology and Measurement. London: Routhledge.

Labrador, F. J., y Villadangos, S. M. (2010). Menores y nuevas tecnologías: conductas indicadoras de posible problema de adicción. Psicothema, 22(2), 180-188.

Lorente, S. (2002). Juventud y teléfonos móviles: algo más que una moda. Estudios de Juventud, 57, 9-24.

Mora, C. (2013). Revisión de pruebas psicológicas para niños validadas o estandarizadas en Colombia. Colombia: S/E.

Morales, L. F. (2012). Adicción al teléfono móvil o celular. Revista de Psicología de Arequipa, 2(1), 94-102.

Muñoz-Rivas, M. J., y Agustín, S. (2005). La adicción al teléfono móvil. Psicología Conductual, 13, 481-493. 
Oliva, A., Hidalgo, M., Moreno C., Jiménez, L., Jiménez, A., Antolín, L., y Ramos, P. (2012). Uso y riesgo de adicciones a las nuevas tecnologías entre adolescentes y jóvenes andaluces. España: Editorial Agua Clara.

Pedrera, I. y Revuelta, F. (2015). Mobile learning: una propuesta de intervención para la igualdad de género en educación secundaria. Revista Qurriculum, 28, 129-143.

Pedrero, E. J., Rodríguez, M. T., y Ruiz, J. M. (2012). Adicción o abuso del teléfono móvil. Revisión de la literatura. Adicciones, 24(2), 139-152.

Quevedo, C., y Ramírez, P. (2012). Adicción a Internet en cibernautas. Revista de Psicología (Universidad Católica de Santa María), 2(2), 5-14.

Pérez-Gil, J., Chacón, S., y Moreno, R. (2000). Validez de constructo: el uso de análisis factorial exploratorio-confirmatorio para obtener evidencias de validez. Psicothema, 12(2), 442-446.
Saldaña, D. (2001). Nuevas tecnologías: nuevos instrumentos y nuevos espacios para la psicología. Apuntes de Psicología, 19(1), 5-10.

Salas, E. (2014). Adicciones psicológicas y los nuevos problemas de salud. Cultura, 28, 111-146.

Salas, E. y Escurra, L. M. (2014). Uso de redes sociales entre estudiantes universitarios limeños. Revista Peruana de Psicología y Trabajo Social, 3(1), 75-90.

Sánchez-Carbonell, X., Beranuy, M., Castellana, M., Chamarro, A., y Oberst, U. (2008). La adicción a Internet y al móvil: ¿moda o trastorno? 20(2), 149-160.

Sandoz, J. (2004). Internet Addiction. Annals of the American Psychotherapy Association, 6, 7-34.

Vargas-Hernández, J. G. (2014). Segment Analysis of Mobile Phones in Mexico: lusacell, 3(10), 465-472. 


\title{
ANEXo 1
}

\section{FICHA TÉCNICA DEL TEST DE DEPENDENCIA AL MÓVIL (TDM)}

\author{
Autor : Mariano Chóliz Montañés \\ Año \\ Procedencia \\ Tiempo de Administración \\ Administración \\ Ámbito de Aplicación \\ Descripción de la prueba \\ : 2010 \\ : España \\ : Aproximadamente 10 minutos. \\ : Individual o colectiva \\ : Edades a partir de los 14 a 33 años \\ : La prueba consta de 22 ítems y cuatro dimensiones
}

Dimensión I: abstinencia (ítems 13, 15, 20, 21 y 22), siendo el puntaje máximo 20.

La dimensión I, abstinencia, es una de las principales características de los trastornos adictivos, que se expresa por un severo malestar provocado cuando no se tiene la posibilidad de utilizar el móvil o hace tiempo que no se puede usar. Igualmente, los problemas de funcionamiento del móvil o las dificultades en su uso generan reacciones emocionales negativas magnificadas.

Dimensión II: abuso y dificultad en controlar el impulso (ítems 1, 4, 5, 6, 8, 9, 11, 16 y 18); el puntaje máximo es 36.

La dimensión II, abuso y dificultad en controlar el impulso, se refiere a la utilización excesiva del móvil en cualquier momento y situación, que está relacionada con una de las características principales de los trastornos adictivos: la dificultad para dejar de consumir, en este caso, para dejar de utilizar el móvil. El uso excesivo tiene mucho que ver con la dificultad de controlar la conducta. Las nuevas aplicaciones del móvil, especialmente las de mensajería (WhatsApp, Line o Viber), dificultan enormemente el control conductual.

Dimensión III: Problemas ocasionados por el uso excesivo (ítems 2, 3, 7 y 10); el puntaje máximo es 16.

La dimensión III, problemas ocasionados por el uso excesivo, evalúa las consecuencias negativas que tiene la utilización excesiva del móvil o su uso inapropiado en la 
esfera personal (gasto excesivo, interferencia con otras actividades importantes...), social (problemas en sus relaciones con los demás) o familiar.

Dimensión IV: Tolerancia (ítems 12, 14, 17 y 19); el puntaje máximo es 16.

Finalmente, la dimensión IV, tolerancia, también es una característica singular de los trastornos adictivos y se refiere a la necesidad de utilizar cada vez más el móvil para conseguir la misma satisfacción o que el uso moderado no llegue a ser suficiente para la persona.

Calificación:

Cada ítem tiene cuatro posibles puntajes en una escala Likert de 0 a 4 , donde las puntuaciones directas oscilan entre 0 y 88 como puntaje máximo.

Cada dimensión obtiene un puntaje de acuerdo al resultado de los ítems correspondientes a dicha dimensión. Posteriormente, se hace la interpretación cualitativa dependiendo del puntaje de cada dimensión. 


\section{ANEXo 2}

Tabla 8

Baremos para la Universidad Nacional de San Agustín

\begin{tabular}{|c|c|c|c|c|}
\hline PERCENTIL & FACTOR I & FACTOR II & FACTOR III & IDM total \\
\hline 5 & 2.00 & 2.00 & .00 & 6.00 \\
\hline 10 & 4.00 & 3.00 & .00 & 9.00 \\
\hline 15 & 5.00 & 4.00 & .00 & 11.00 \\
\hline 20 & 7.00 & 4.00 & .00 & 13.00 \\
\hline 25 & 8.00 & 5.00 & .00 & 16.00 \\
\hline 30 & 10.00 & 6.00 & 1.00 & 19.00 \\
\hline 35 & 11.00 & 7.00 & 1.00 & 21.00 \\
\hline 40 & 13.00 & 8.00 & 1.00 & 23.00 \\
\hline 45 & 14.00 & 9.00 & 1.00 & 25.75 \\
\hline 50 & 16.00 & 9.00 & 2.00 & 27.50 \\
\hline 55 & 18.00 & 10.00 & 2.00 & 30.00 \\
\hline 60 & 19.00 & 11.00 & 2.00 & 32.00 \\
\hline 65 & 21.00 & 12.00 & 3.00 & 34.00 \\
\hline 70 & 23.00 & 12.00 & 3.00 & 37.00 \\
\hline 75 & 25.00 & 14.00 & 3.00 & 40.00 \\
\hline 80 & 27.00 & 15.00 & 4.00 & 44.00 \\
\hline 85 & 28.00 & 16.00 & 5.00 & 48.00 \\
\hline 90 & 31.00 & 18.00 & 6.00 & 52.00 \\
\hline 95 & 34.00 & 21.00 & 7.00 & 57.00 \\
\hline Media & 16.82 & 9.89 & 2.29 & 28.99 \\
\hline Desv. típ. & 10.196 & 5.855 & 2.412 & 16.196 \\
\hline
\end{tabular}




\section{ANEXO 3}

Tabla 9.

Baremos para la Universidad Católica San Pablo

\begin{tabular}{|c|c|c|c|c|}
\hline PERCENTIL & FACTOR I & FACTOR II & FACTOR III & IDM total \\
\hline 5 & 5 & 4.00 & 2.00 & .00 \\
\hline 10 & 10 & 6.00 & 5.00 & .00 \\
\hline 15 & 15 & 9.00 & 6.00 & .00 \\
\hline 20 & 20 & 11.00 & 7.00 & 1.00 \\
\hline 25 & 25 & 12.00 & 8.00 & 1.00 \\
\hline 30 & 30 & 14.80 & 9.00 & 1.00 \\
\hline 35 & 35 & 17.00 & 10.00 & 2.00 \\
\hline 40 & 40 & 18.40 & 11.00 & 2.00 \\
\hline 45 & 45 & 20.00 & 12.00 & 3.00 \\
\hline 50 & 50 & 21.00 & 12.00 & 3.00 \\
\hline 55 & 55 & 22.00 & 13.00 & 3.00 \\
\hline 60 & 60 & 24.00 & 14.00 & 4.00 \\
\hline 65 & 65 & 25.00 & 14.00 & 4.00 \\
\hline 70 & 70 & 26.00 & 15.00 & 5.00 \\
\hline 75 & 75 & 27.00 & 16.00 & 6.00 \\
\hline 80 & 80 & 29.00 & 17.00 & 6.00 \\
\hline 85 & 85 & 31.00 & 19.00 & 7.00 \\
\hline 90 & 90 & 34.40 & 21.00 & 7.40 \\
\hline 95 & 95 & 38.00 & 23.00 & 9.00 \\
\hline Media & 20,64 & 12.56 & 3.45 & 36.65 \\
\hline Desv. típ. & 10,287 & 5.991 & 2.944 & 17.019 \\
\hline
\end{tabular}

